\title{
Development of an HPLC method \\ for the determination of phenolic by-products: optimisation of the separation by means of the experimental designs methodology
}

\author{
B. Motamed ${ }^{1}$, J.-L. Böhm ${ }^{1}$, D. Hennequin ${ }^{1}$, H. Texier ${ }^{2}$, R. Mosrati $^{1}$ and D. Barillier ${ }^{1 *}$ \\ ${ }^{1}$ Équipe de recherche en Physico-chimie et Biotechnologies, IUT-UFR Sciences, Boulevard du Maréchal Juin, 14032 Caen, France \\ ${ }^{2}$ Laboratoire de Morphodynamique Continentale et Côtière, Université de CAEN, Avenue des Tilleuls, 14032 Caen, France
} realized by Reverse Phase HPLC using a binary gradient water-acetic acid/acetonitrile whose elution program was optimized by means of the experimental design methodology.

Significant factors are: the initial isocratic elution time, the gradient running time and the gradient curvature. Modelization was made by using a central composite plane in 17 experiments. Responses were measured by the resolution between four couples of components: p-hydroxybenzoic acid - phenol, phenol - p-hydroxybenzaldehyde, acetovanilone - acetosyringone and acetosyringone - benzoic acid.

According to the resulting quatradic model, an optimized program of gradient elution was set up and successfully tested as it gives satisfactory separations (isocratic elution time: $8 \mathrm{~min}$; gradient running time from $1 \%$ to $25 \%$ acetonitrile: $40 \mathrm{~min}$; linear gradient; room temperature).
The separation of nine phenolic by-products was

The analysis of this kind of phenolic compound mixtures has been widely developed in wood chemistry and biochemistry as well as in food controls, in soil analyses (humus) and more generally in environment pollution examinations (natural or waste-water). First attempts have been achieved by thin layer chromatography [2-6] or by gaz chromatography in the 60s requiring a previous derivatization of the compounds [7-13]. High performance liquid chromatography was then used in the 70 s and 80 s. So were described, according to the stationary phase being used, applications by ion-exchange chromatography [14-16], by ion-pair chromatography $[17,18]$ or by partition chromatography on various normal and reverse-phases [19-35]. Among these HPLC methodologies, partition on reverse-phase generally emerges as being the most convenient technique, using octadecyl silica-bonded columns as hydrophobic stationary phases in conjunction with polar methanol or acetonitrile/water mixtures in acidic medium as mobile phases.

Focussing on the mixture that we had to investigate, it can be seen that some compounds differ widely in their polarity and hydrophobia-hydrophily properties according to their various functionalities: phenol(s), ether, aldehyde, ketone and/or carboxylic acid. Thus, to achieve a good

Table I. Phenolic compounds investigated by HPLC and order of elution.

\section{Compound} Peak $n^{\circ}$

The foundry sands, reused in civil engineering (for the real-
isation of road undercoats), have to respect a content lower than $5 \mathrm{mg} / \mathrm{kg}$ in leachatable phenols to be valued.

During the study of a biodegradation process of phenolic compounds held in foundry sands, we had to separate and quantify a certain number of phenolic by-products selected as potential markers of the process monitoring.

Phenolic by-products to be analyzed are collected in the table I: they are aromatic compounds resulting from the cupric oxidation in basic medium at $170{ }^{\circ} \mathrm{C}$ of sand leachate followed by acidification and liquid-liquid extraction of the oxidation products with ethyl acetate [1].

\begin{tabular}{ll}
\hline 3,4,5-trihydroxybenzoic acid (gallic acid) & 1 \\
4-hydroxybenzoic acid & 2 \\
phenol & 3 \\
4-hydroxybenzaldehyde & 4 \\
4-hydroxy-3-methoxybenzaldehyde (vanillin) & 5 \\
4-hydroxy-3-methoxyacetophenone (acetovanillone) & 6 \\
4-hydroxy-3,5-dimethoxyacetophenone & \\
(acetosyringone) & 7 \\
benzoic acid & 8 \\
4-methoxybenzoic acid (anisic acid) & 9
\end{tabular}


separation by reversed-phase chromatography, one must rely on an efficient stationary phase as well as must realise a very selective gradient of mobile phase assuming that the solvent strength must be increased in a continuous manner without any decrease in the selectivity. These problems can be solved by starting the elution with a very hydrophilic solvent in order to improve the separation of the earliest very polar polyphenols (gallic acid) and the polar monophenols (phenol and $p$-hydroxybenzoic acid). Then the solvent strength must increase gradually towards a less hydrophilic nature in order to separate phenolic aldehydes ( $p$-hydroxybenzaldehyde, vanillin), phenolic ketones-ethers (acetovanillone, acetosyringone) and lastly the non phenolic carboxylic acids (benzoic and anisic acids).

The strong evolution of the eluent composition during the chromatography will not on the other hand improve the resolution between two contiguous compounds of closely similar properties and that is the case for some compounds constituting the previous couples.

Faced with such a difficult separation we were inclined to duplicate one of the described methodologies closest to our objective. Among numerous methods one can take out:

- the separation by Kögel et al. [22] of lignin derived products such as acid and phenolic aldehydes of benzoïc and cinnamic series requiring a concave binary gradient elution achieved with an acetonitrile/buffer phosphate mixture at room temperature;

- the more systematic study by Burtscher et al. [23] of the separation of almost thirty phenols, benzenic ethers, benzaldehydes, acetophenones and benzoic and cinnamic acids using a binary gradient acetonitrile/phosphate buffer as eluent at $50{ }^{\circ} \mathrm{C}$;

- the separation by Charrière et al. [35] of about twenty phenolic compounds using a very complex binary gradient elution based on a acetonitrile/water/acetic acid mixture at $37{ }^{\circ} \mathrm{C}$.

Therefore when attempting to closely reproduce the results described in the previous methodologies we were confronted with a lot of problems that are well known to chromatographists:

- some gradient elution programs, as the one used by Charrière et al. [35], are so complex that the experiments are extremely difficult to reproduce if the know-how and the equipment of the original laboratory are not available;

- other gradients, as the one used by Burtscher et al. [23], are simpler (two-slope linear gradient) but lead in our case to poor separations, particularly in the acetovanillone-acetosyrigone-benzoic acid case;

- described methods generally refer to a type of stationary phase (octadecyl C18) corresponding to commercial packing materials of particular trademarks, varying from one methodology to another. However one in the laboratory does not necessarily have the same trademarked column but one from other suppliers which can differ slightly by its hydrophobicity, its granulometry and shape of the base silica, the ratio of residual silanols, even by the size and packing process of the column, etc. It may thus be impossible to translate a separation on a given commercial stationary phase to another similar phase from another manufacturer.

Lastly if it is easy to reproduce a simple separation (isocratic for example) with equipment different from one laboratory to another it is rather hazardous in the case of such a delicate separation as the one that is proposed to us. Moreover it should be also mentioned, as the method is aimed at checking the processing of a low-added value product of civil engineering, that our objective should be to find the easiest methodology relying upon basic parameters, equipment and technologies: elution at room temperature, available columns routinely used in the laboratory and elution gradient as simple as possible.

That is why, after having failed to duplicate some of the above-mentioned separations, we were forced to develop our own chromatographic conditions.

\section{Experimental}

\section{Apparatus}

The HPLC system was equipped with two Waters M510 pumps allowing the obtention of a high-pressure gradient programmed by a Maxima Baseline software under Windows ${ }^{\mathrm{TM}}$. The sample was injected by a sample loop valve fitted with a $20 \mu \mathrm{L}$ loop (Reodyne 7010). The detector was a Waters UV486 spectrophotomer set at $275 \mathrm{~nm}$. The signal was treated with either a ChromJet integrator (from TSP), or the Maxima Baseline Waters software under Windows $^{\mathrm{TM}}$ on a AST Bravo MS 4/66d microcomputer.

\section{Column}

We used a $25 \mathrm{~cm} \times 3.9 \mathrm{~mm}$ i.d. steel column filled with $\mu$ Bondapak C18, $10 \mu \mathrm{m}$ (from Waters) protected by a short $2 \mathrm{~cm} \times 3.9 \mathrm{~mm}$ i.d. steel pre-column filled with the same stationary phase and placed immediately before the main column. The column was thermostated at room temperature by a simple draught proofing in a box filled with a thermal insulating.

\section{Eluents and standards}

Gradient elution was carried out at a flow rate of $0.8 \mathrm{~mL} . \mathrm{min}$. The starting eluant was A: ultra pure water (obtained by ultra-filtration on a Milli $\mathrm{Q}^{+}$system from Millipore) $96 \%$; acetic acid R.P. (Carlo Erba) $3 \%$; acetonitrile HPLC grade (Carlo Erba) $1 \%$. The finishing eluent was B: ultra pure water $72 \%$; acetic acid $3 \%$; acetonitrile $25 \%$. The gradient started from $100 \%$ of A to reach $100 \%$ of B according to the program of the experiment (see table II). The acetonitrile content was maintained for $10 \mathrm{~min}$ 
Table II. Matrix of the experiments of the centred composite plane. Responses in Resolution (Rs).

\begin{tabular}{|c|c|c|c|c|c|c|c|c|}
\hline Entry & Run order & Factor $A$ & Factor $B$ & Factor $C$ & $\begin{array}{c}\text { Response } \\
R s_{1}\end{array}$ & $\begin{array}{c}\text { Response } \\
R s_{2}\end{array}$ & $\begin{array}{c}\text { Response } \\
R s_{3}\end{array}$ & $\begin{array}{c}\text { Response } \\
R s_{4}\end{array}$ \\
\hline 1 & 16 & -1 & -1 & -1 & 1.39 & 1.22 & 0.69 & 1.59 \\
\hline 2 & 3 & +1 & -1 & -1 & 0 & 2.50 & 0.88 & 1.26 \\
\hline 3 & 14 & -1 & +1 & -1 & 0 & 2.48 & 1.14 & 0.93 \\
\hline 4 & 15 & +1 & +1 & -1 & 0.78 & 3.41 & 1.08 & 0.55 \\
\hline 5 & 17 & -1 & -1 & +1 & 3.18 & 1.21 & 0.85 & 3.05 \\
\hline 6 & 1 & +1 & -1 & +1 & 2.52 & 0 & 0.92 & 2.32 \\
\hline 7 & 4 & -1 & +1 & +1 & 4.29 & 0.87 & 0.89 & 3.18 \\
\hline 8 & 12 & +1 & +1 & +1 & 2.48 & 0 & 0.98 & 2.08 \\
\hline 9 & 5 & -1.68 & 0 & 0 & 2.78 & 0 & 1.30 & 1.43 \\
\hline 10 & 8 & +1.68 & 0 & 0 & 0.68 & 1.92 & 1.36 & 1.27 \\
\hline 11 & 7 & 0 & -1.68 & 0 & 2.27 & 0 & 0.92 & 2.08 \\
\hline 12 & 6 & 0 & +1.68 & 0 & 1.06 & 1.61 & 1.57 & 0.88 \\
\hline 13 & 11 & 0 & 0 & -1.68 & 2.18 & 2.60 & 0.60 & 1.35 \\
\hline 14 & 9 & 0 & 0 & +1.68 & 3.68 & 1.60 & 0 & 2.70 \\
\hline 15 & 2 & 0 & 0 & 0 & 1.72 & 1.00 & 1.42 & 1.33 \\
\hline 16 & 10 & 0 & 0 & 0 & 1.62 & 1.02 & 1.35 & 1.26 \\
\hline 17 & 13 & 0 & 0 & 0 & 1.86 & 0.98 & 1.53 & 1.37 \\
\hline
\end{tabular}

\begin{tabular}{lccc}
\hline Levels & $\begin{array}{c}\text { Factor } A \\
\text { Isocratic elution time } \\
(\text { min) }\end{array}$ & $\begin{array}{c}\text { Factor } B \\
\text { Gradient running time } \\
(\text { min) }\end{array}$ & $\begin{array}{c}\text { Factor C } \\
\text { Gradient curvature }\end{array}$ \\
\hline-1.68 & 0 & 30 & -8 \\
-1 & 2 & 35 & -5 \\
0 & 5 & 40 & 0 \\
+1 & 8 & 45 & +5 \\
+1.68 & 10 & 50 & +8 \\
\hline
\end{tabular}

after the end of the separation (corresponding to the peak of anisic acid) and then the column was re-equilibrated back to the initial conditions (100\% of A). They were adjusted by a 1 min step-gradient which was held for 15 min before the next injection.

A standard solution of the mixed phenolic compounds was prepared by dissolving $10 \mathrm{mg}$ of each component (Tab. I) in $100 \mathrm{~mL}$ of acetonitrile HPLC grade. Diluted standards were prepared from the previous one in ultra-pure water.

\section{Selectivity and resolution}

Selectivities are stated as the ratio of the retention times recorded within $\pm 1 / 100 \mathrm{~min}$ and are assessed at $\pm 0.1 \%$. Resolutions are calculated on the basis of the ratio between differences in retention times and half-band widths measured manually within $\pm 1 / 2 \mathrm{~mm}$ on the chromatogram reprocessed with a chart speed of $4 \mathrm{~cm} / \mathrm{min}$. They are assessed at $\pm 1 \%$.

\section{Results and discussion}

The basic parameters of the chromatographic method were chosen according to the data of the literature and particularly those applied by Charrière et al. [35]. Then we used:

- a $\mu$ Bondapak C18 column of octadecyl silica-bonded type, with usual granulometry of $10 \mu \mathrm{m}$, irregular particle shape, pore size of $125 \mathrm{~A}^{\circ}$ and a geometry of $3,9 \times$ $250 \mathrm{~mm}$;

- an eluent buffered at a $\mathrm{pH}$ close to 3 by addition of acetic acid to minimize the ionization of carboxylic compounds and fix them under the major protonated form;

- an eluent composition set at a $1 \%$ ratio of acetonitrile (eluent A) at the beginning of chromatography in agreement with the elution of the most hydrophilic compound (gallic acid); 
- an eluent composition set at a $25 \%$ ratio of acetonitrile (eluent B) at the end of chromatography, adapted to the elution of the most hydrophobic compound (anisic acid);

- a flow-rate of $0.8 \mathrm{~mL}$.min which was determined as optimal when the apparent height equivalent to a theoretical plate $(\mathrm{H})$ was plotted versus the linear velocity of the eluent $(\mu)$.

\section{Experimental design}

Our preliminary works, and so our failure to partially reproduce the methods of the literature, showed that the difficulties of the separation concern essentially the $p$-hydroxybenzoic acid, phenol and $p$-hydroxybenzaldehyde on the one hand, acetovanillone, acetosyringone and benzoic acid on the other hand. So we tried to optimise the chromatographic separation between these two series of products taking into account the fact that, without exceptions, the eluting order of components is invariable and we chose to measure separations between the four following couples:

- $p$-hydroxybenzoic acid and phenol (response 1);

- phenol and $p$-hydroxybenzaldehyde (response 2);

- acetovanillone and acetosyringone (response 3);

- acetosyringone and benzoic acid (response 4).

Recorded responses could be determined either by the resolution or either by the selectivity. Although selectivity could be evaluated more precisely and easily than resolution, the calculation of a selectivity does not permit one to know a priori if two compounds are separated in chromatography. A selectivity at above 1 enables one only to say that they are in theory separable. In the last, responses were given in resolution that is more representative to quantify the separation between to contiguous peaks.

Experimental design consists in modelling and optimising the program of elution corresponding to a binary gradient. Considering that this gradient must evolve from the pure eluent $\mathrm{A}$ at the beginning of chromatography to the pure eluent $\mathrm{B}$ at the end of chromatography, factors taken into account in the design were the following ones:

- the isocratic elution time (factor A), set inside the domain between 0 and $10 \mathrm{~min}$;

- the gradient running time from $0 \%$ to $100 \%$ of eluent B (factor B) set between 30 and $50 \mathrm{~min}$;

- the mathematical curvature of the gradient (factor $\mathrm{C}$ ): concave, linear or convex fixed between -8 and +8 .

The limits of every domain were set according to the data of preliminary experiments and the equipment abilities. The analysis of data was carried out by means of the Modde 4.0 software (from Umetri).

The modellisation was led by a central composite design. The matrix of experiments was built on the basis of the eight experiments of the $2^{3}$ design, six experiments of star points and three experiments in the centre of the domain as quoted in table II. The chosen central composite design satisfies the standard of isovariance by rotation. Star points of the design are situated at the distance $\pm \alpha$ of the domain center. The calculation of the $\alpha$ value does not depend of the number of centre points. A minimal number of three central points was proposed by the Modde 4.0 software and this one was adopted in order to minimise the number of attempts of the heavy experimental work.

Experiments were recorded at random to avoid the block effects. Data of the 17 chromatographic runs are collected in table III including resolution and selectivity between contiguous peaks. They show that, none of the proposed elution programs could be directly applied for a separation of the nine products outside of the experiments in the centre of the plane.

The percentage of variance explained by the mathematical model $\left(R^{2}\right)$ compared with the variance of the experimental error for the four responses in resolution is given in table IV. The validity of the models seems satisfactory for the set of responses in regard of $R^{2}$ values upper than $90 \%$. The coefficients of quadratic models calculated for the four responses in resolution are given in table IV. The determination of significant factors and interactions is realized from the probability attached to the value of the coefficient at the confidence level of $95 \%$. The effect is significant if this probability is lower than 0.05 . So effects of factors $A, B$ and $C$ seem to be significant. Only interactions $C * C$ and $A * C$ are significant for responses $R s_{2}$ and $R s_{3}$.

The other not significant interactions for the set of responses are eliminated in the calculation of the terms of quadratic models to improve the fitting rate. This one is then given by the $Q^{2}$ parameter (typical of the Modde software) which is the variation of the response than can be predicted by the model. $Q^{2}$ is an underestimated measure of the goodness of fit of the model, while $R^{2}$ is the fraction of variation of the response explained by the model and represents an overestimated measure. $Q^{2}$ values found for $R s_{1}$ to $R s_{4}$ are respectively $0.61,0.54,0.80$ and 0.57 .

\section{Optimisation}

Optimisation is attempted to determine the best combinations of the three factors allowing a good separation of phenolic compounds from models elaborated for responses in resolution. The conditions of optimisation consist in fixing, for every response $R s_{1}$ to $R s_{4}$, the minimal value of resolution corresponding to a satisfactory separation of two peaks. It is usually considered that two compounds are separated if $R s \geq 1.2$ when measured with two symmetrical peaks. Here we preferred to set empirically a minimal value for every couple of peaks, determined a posteriori after examining the various chromatograms of the plan (given in table III) and when checking that the separations between two peaks seems quite effective. The values (indicated $R s_{1}$ to $R s_{4}$ ) can differ from one couple to another because of the relative asymmetry of the various peaks. The resolution target values thus determined are reliable but entail a great uncertainty considering that measures are achieved manually on the chromatogram within $\pm 1 / 2 \mathrm{~mm}$. 
Table III. Retention times ( $t r$ in $\min$ ), Selectivities $(\alpha)$ and Resolutions $(R s)$ in the 19 chromatograms resulting of the experimental design and optimization.

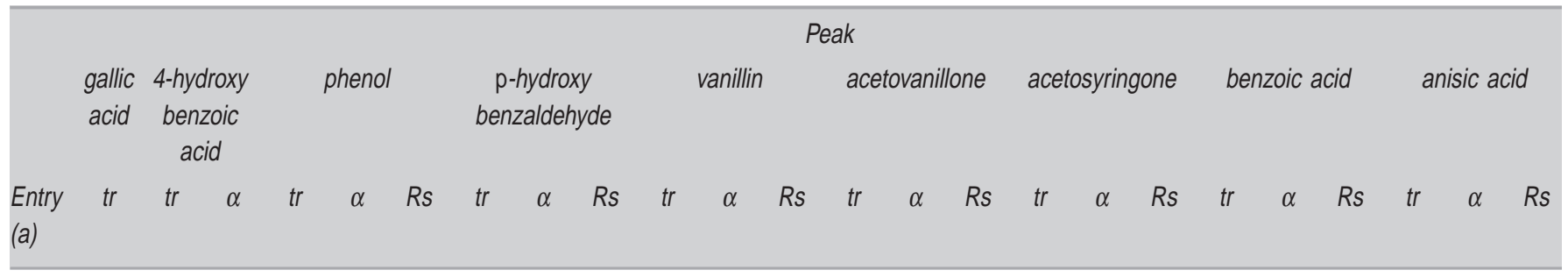

$1 \quad 19.2034 .961 .8236 .911 .0561 .3938 .561 .0451 .22 \quad 42.061 .0913 .2043 .961 .0452 .1244 .651 .016 \quad 0.6946 .301 .037 \quad 1.59 \quad 50.491 .0904 .20$

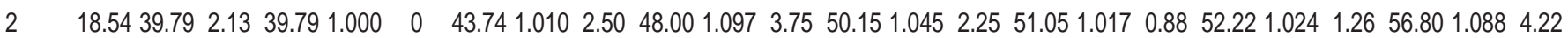

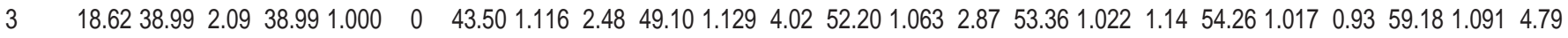
$4 \quad 18.3542 .002 .2843 .341 .032 \quad 0.7848 .381 .1163 .41 \quad 54.741 .1314 .4058 .061 .0612 .6759 .321 .0221 .08 \quad 59.861 .0090 .55 \quad 64.971 .0854 .64$

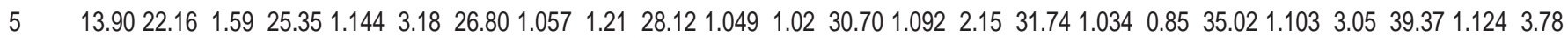

$6 \quad 17.5027 .261 .55 \quad 30.481 .1182 .5230 .481 .000 \quad 0 \quad 33.661 .1042 .35 \quad 36.401 .0812 .2937 .561 .032 \quad 0.92 \quad 40.361 .075 \quad 2.3245 .041 .116 \quad 4.12$

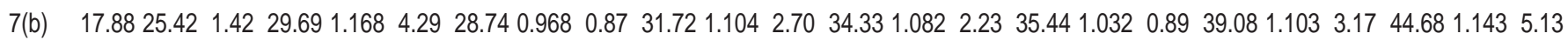
$8 \quad 18.0628 .991 .60 \quad 32.401 .1182 .4832 .401 .000 \quad 0 \quad 36.241 .1192 .5939 .281 .0832 .4840 .701 .036 \quad 0.98 \quad 43.411 .067 \quad 2.0849 .861 .149 \quad 5.16$ $9 \quad 14.9927 .401 .82 \quad 31.161 .1372 .7831 .161 .000 \quad 0 \quad 35.501 .1393 .0738 .941 .0972 .9040 .511 .0401 .3042 .601 .0591 .4347 .931 .1254 .12$ $10 \quad 19.3636 .161 .86 \quad 37.151 .027 \quad 0.6840 .141 .0801 .9245 .331 .1293 .9149 .041 .0822 .9850 .701 .0341 .36 \quad 52.301 .0321 .27 \quad 57.971 .108 \quad 4.69$

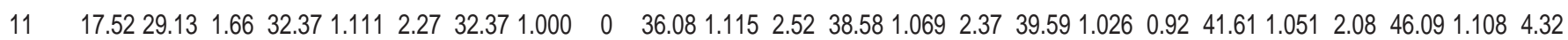
$12 \quad 18.1933 .881 .86 \quad 35.571 .0501 .06 \quad 38.301 .077 \quad 1.6144 .241 .1553 .88 \quad 48.621 .0993 .1150 .881 .046 \quad 1.57 \quad 52.161 .025 \quad 0.88 \quad 58.701 .125 \quad 6.20$ $13 \quad 18.1144 .112 .43 \quad 47.791 .0832 .1850 .701 .0612 .6053 .501 .0552 .98 \quad 55.151 .0311 .5955 .741 .011 \quad 0.60 \quad 56.961 .022 \quad 1.35 \quad 61.591 .081 \quad 4.41$ 14(c) $14.8220 .701 .3925 .191 .2173 .6823 .21 \quad 0.921 \quad 1.6025 .191 .0851 .6026 .881 .0671 .1126 .881 .000 \quad 0 \quad 30.331 .1282 .7034 .051 .123 \quad 3.33$ $15 \quad 17.2831 .161 .8033 .541 .0761 .7235 .121 .0471 .0040 .001 .1393 .6343 .651 .0912 .7945 .381 .0401 .4246 .941 .0341 .33 \quad 52.291 .1144 .50$ $16 \quad 17.4431 .421 .80 \quad 33.701 .073 \quad 1.6235 .371 .0501 .0240 .281 .1393 .6243 .931 .0912 .6345 .691 .040 \quad 1.3547 .201 .0331 .26 \quad 52.561 .114 \quad 4.23$

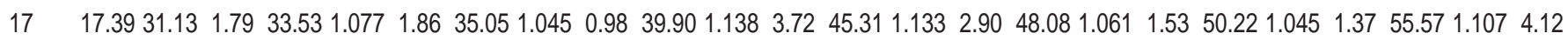
$18 \quad 17.8432 .531 .82 \quad 33.761 .038 \quad 0.90 \quad 36.531 .0821 .8041 .881 .1463 .7045 .741 .092 \quad 2.8047 .811 .045 \quad 1.55 \quad 49.001 .025 \quad 0.99 \quad 55.041 .123 \quad 4.90$ $19 \quad 17.3432 .261 .8633 .531 .0390 .9236 .321 .0231 .8041 .731 .1493 .7045 .581 .092 \quad 3.1047 .441 .041 \quad 1.5548 .451 .021 \quad 0.98 \quad 53.861 .1124 .39$

(a) Entries 1 to 17 correspond to the experiments refered in the table II. Entries 18 and 19 correspond to the experiments of optimized programs $n^{\circ} 1$ and $n^{\circ} 2$ respectively. Selectivity and resolution are related for every compounds to the preceding one in the table except (b) phenol is eluted after $p$-hydroxybenzaldehyde (c) phenol is eluted after $p$-hydroxybenzaldehyde and coeluted with vanillin.

Table IV. Coefficients of quadratic models for responses in resolution $\boldsymbol{R} \boldsymbol{s}_{1}$ to $\boldsymbol{R} \boldsymbol{s}_{4}$. Probabilities at the confidence level 0.95 (significant factors for a probability lower than 0.05 are indicated in bold type) and validity of the model. Isocratic elution time: factor A ; gradient time: factor B ; gradient curvature: factor C.

\begin{tabular}{|c|c|c|c|c|c|c|c|c|}
\hline & \multicolumn{2}{|c|}{$R s_{1}$} & \multicolumn{2}{|c|}{$R s_{2}$} & \multicolumn{2}{|c|}{$R s_{3}$} & \multicolumn{2}{|c|}{$R s_{4}$} \\
\hline & Coefficient & Probability & Coefficient & Probability & Coefficient & Probability & Coefficient & Probability \\
\hline Constant & 1.733 & $2.50610^{-3}$ & 1.000 & 0.012 & 1.433 & $7.71410^{-7}$ & 1.320 & $7.56610^{-4}$ \\
\hline$A$ & -0.485 & 0.025 & 0.246 & 0.112 & 0.029 & 0.406 & -0.207 & 0.081 \\
\hline B & -0.123 & 0.448 & 0.316 & 0.041 & 0.128 & $4.96110^{-3}$ & -0.243 & 0.037 \\
\hline C & 1.258 & $7.6210^{-4}$ & -0.886 & $1.54310^{-3}$ & -0.042 & 0.323 & 0.704 & $1.13310^{-3}$ \\
\hline$A A$ & 0.023 & 0.906 & -0.060 & 0.706 & -0.018 & 0.653 & 0.080 & 0.508 \\
\hline BB & -0.005 & 0.970 & -0.071 & 0.538 & -0.038 & 0.205 & 0.074 & 0.400 \\
\hline $\mathrm{CC}$ & 0.032 & 0.899 & 0.680 & 0.012 & -0.487 & $4.62610^{-5}$ & 0.263 & 0.118 \\
\hline$A B$ & 0.127 & 0.572 & $-1.2510^{-3}$ & 0.994 & -0.029 & 0.518 & -0.053 & 0.697 \\
\hline$A C$ & -0.233 & 0.317 & -0.536 & 0.021 & $3.7510^{-3}$ & 0.932 & -0.140 & 0.318 \\
\hline $\mathrm{BC}$ & 0.210 & 0.363 & -0.314 & 0.118 & -0.069 & 0.152 & 0.157 & 0.267 \\
\hline
\end{tabular}

Validity of the model

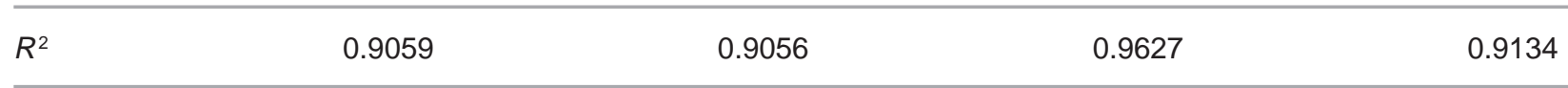


Table V. Optimization experiments: comparison between experimental and theoretical values in resolution.

\begin{tabular}{lcc}
\hline & Experimental values & Values calculated by means of models \\
\hline Gradient program n' 1 (linear) : & $R s_{1}: 0.90$ & $R s_{1}: 1.23$ \\
$A=8$ min $; B=40$ min $; C=0$ & $R s_{2}: 1.82$ & $R s_{2}: 1.13$ \\
& $R s_{3}: 1.57$ & $R s_{3}: 1.40$ \\
Gradient program n 2 (concave) : & $R s_{4}: 0.99$ & $R s_{4}: 1.26$ \\
$A=6$ min $; B=35$ min $; C=-2$ & $R s_{1}: 0.92$ & $R s_{1}: 1.20$ \\
& $R s_{2}: 1.79$ & $R s_{2}: 1.22$ \\
$R s_{3}: 1.20$ \\
$R s_{4}: 1.40$
\end{tabular}

These target values set for every response are close to the values usually quoted for a satisfactory separation; they are the following ones given at $\pm 1 \%$ :

$-R s_{1}=1.40$ ( $p$-hydroxybenzoic acid and phenol);

$-R s_{2}=1.25$ (phenol and $p$-hydroxybenzaldehyde);

- $R s_{3}=1.40$ (acetovanillone and acetosyringone);

- $R s_{4}=1.45$ (acetosyringone and benzoic acid).

Then the Modde 4.0 software generates simplex runs with the differents models. The software searches by successive iterations the best combinations for $A, B$ and $C$ factors which satisfy the target values of responses $R s_{1}$ to $R s_{4}$. Thus two optimal combinations were proposed in the calculation of optimisation: (i) program $n^{\circ} 1$ : isocratic elution time (factor $A$ ) fixed to $8 \mathrm{~min}$, gradient running time (factor $B$ ) 40 min and linear gradient (factor $C=0$ ); (ii) program $n^{\circ} 2$ : isocratic elution time $(A)$ fixed to $6 \mathrm{~min}$, gradient running time $(B) 35 \mathrm{~min}$ and concave gradient $(C=-2)$.

\section{Validation}

These two optimal combinations (linear and concave gradient) were tested experimentally and the results are given in table III (entry 18 and 19). Responses obtained in resolution as well as those calculated by models are collected in the table V.

This optimisation is globally satisfactory as the analysis of chromatograms represented in figure 1 and figure 2 shows that peaks are adequately separated with the two combinations. However examination of the data suggests the following remarks:

- Deviations between theoretical and observed values of resolution are significant. Thus with regard to the theory the $p$-hydroxybenzoic acid/phenol $\left(R s_{1}\right)$ and acetosyringone/benzoic acid $\left(R s_{4}\right)$ couples are less well separated while the phenol/p-hydroxybenzaldehyde $\left(R s_{2}\right)$ and acetovanillone/acetosyringone $\left(R s_{3}\right)$ couples are better separated. This can be explained by the imprecision introduced in the model by uncertainty in the graphic measure of resolutions particularly for the lower values ( $\mathrm{Rs}<0.8)$.

Table VI. Repeated retention times relative to p-anisic acid (mean values of five replicates) and relative standard deviation (RSD).

\begin{tabular}{lcccccc}
\hline & $\operatorname{tr}_{1}$ & $\operatorname{tr}_{2}$ & $\operatorname{tr}_{3}$ & $\operatorname{tr}_{4}$ & $\operatorname{tr}_{5}$ & $R S D(\%)$ \\
\hline gallic acid & 0.243 & 0.243 & 0.242 & 0.238 & 0.234 & 1.59 \\
4-hydroxybenzoic acid & 0.580 & 0.574 & 0.576 & 0.573 & 0.570 & 0.64 \\
phenol & 0.619 & 0.609 & 0.614 & 0.614 & 0.610 & 0.63 \\
4-hydroxybenzaldehyde & 0.663 & 0.655 & 0.658 & 0.659 & 0.654 & 0.54 \\
vanillin & 0.769 & 0.761 & 0.763 & 0.764 & 0.764 & 0.39 \\
acetovanillone & 0.839 & 0.833 & 0.835 & 0.836 & 0.834 & 0.29 \\
acetosyringone & 0.874 & 0.871 & 0.872 & 0.874 & 0.868 & 0.27 \\
benzoic acid & 0.904 & 0.899 & 0.902 & 0.903 & 0.895 & 0.43 \\
anisic acid & 1 & 1 & 1 & 1 & 0 & 1 \\
\hline
\end{tabular}




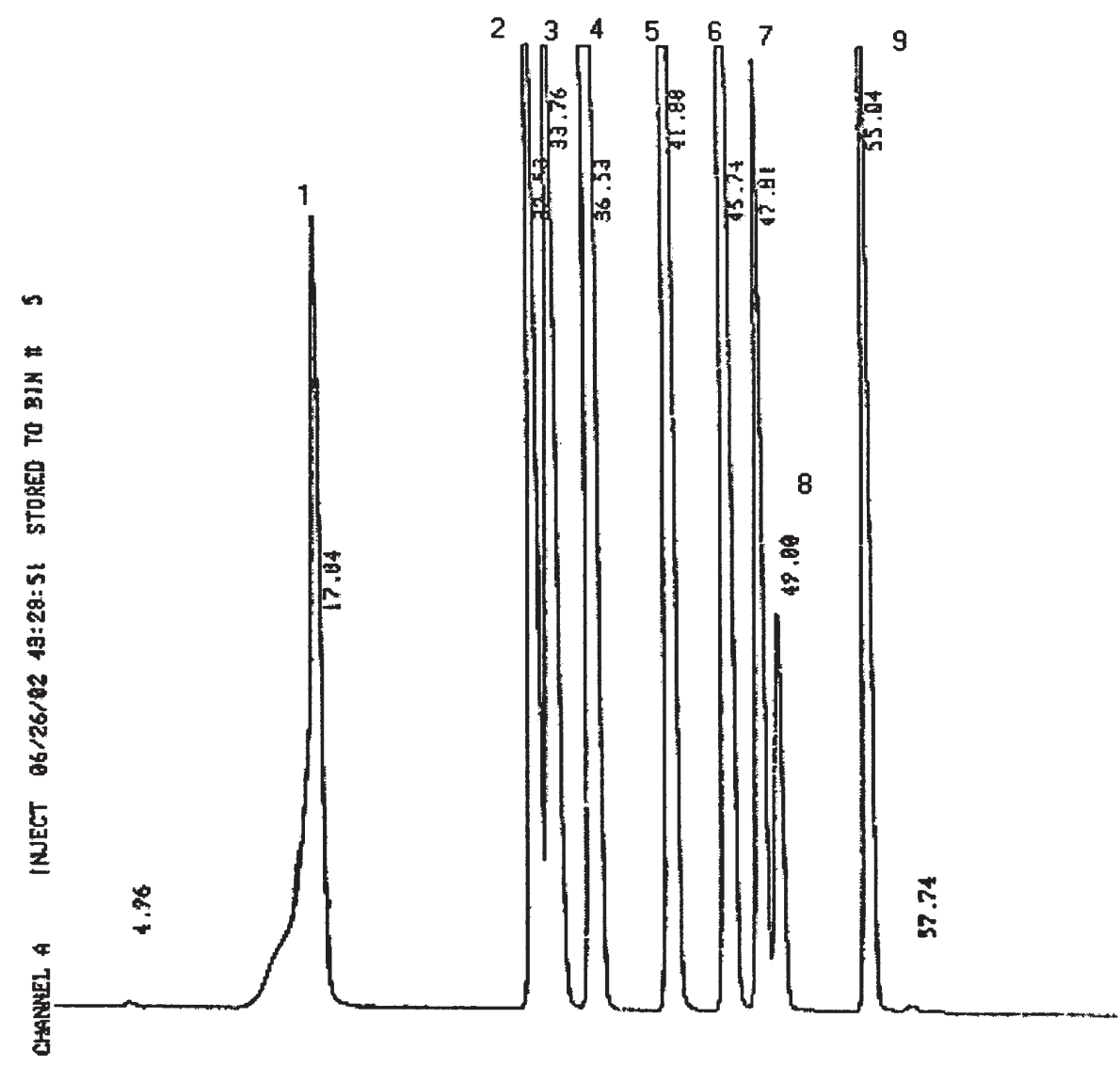

Figure 1. Chromatogram of the separation of nine phenolic compounds.

Elution with optimized gradient $\mathrm{n}^{\circ} 1$ $(A=8 \mathrm{~min} ; B=40 \mathrm{~min} ; C=0) .1$ : gallic acid; 2: $p$-hydroxybenzoic acid; 3: phenol; 4: $p$-hydroxybenzaldehyde; 5: vanillin; 6: acetovanillone; 7 : acetosyringone; 8: benzoic acid; 9: anisic acid.

Figure 2. Chromatogram of the separation of the nine phenolic compounds.

Elution with optimized gradient $\mathrm{n}^{\circ} 2(A=6 \mathrm{~min}$; $B=35 \mathrm{~min} ; C=-2)$. For order of elution see figure 1 .

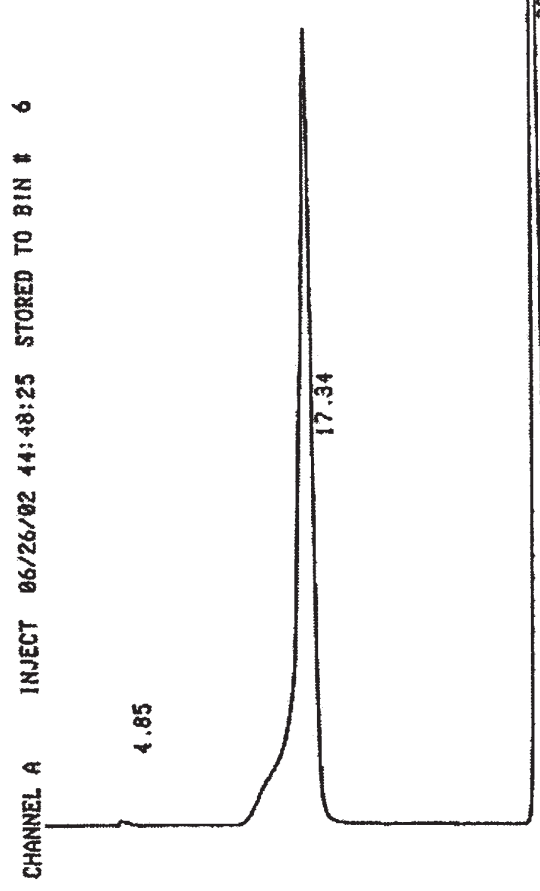

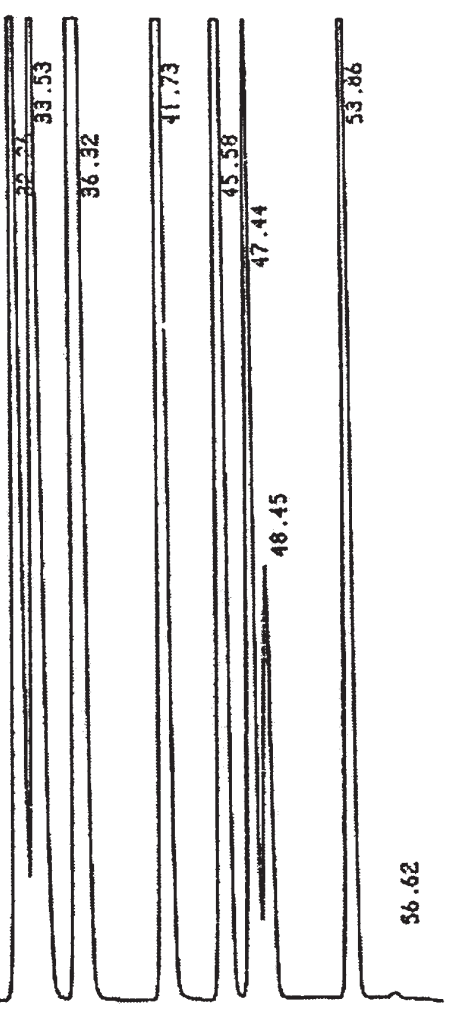

ANALUSIS, 2000, 28, $N^{\circ} 7$ (c) EDP Sciences, Wiley-VCH 2000 
Moreover the zero values assumed for fused peaks are not necessarily right. These values could theoretically be corrected by measurements based on single-product chromatograms but the method is uncertain owing to the poor reproducibility of absolute retention times under such circumstances (elution with complex gradient and retention times closely related one to the other).

- Besides one can think that it is difficult to obtain an ideal solution in that the four responses are not independent of each other because one compares six peaks taken by pairs.

The optimised program $n^{\circ} 1$ (isocratic elution time set to $8 \mathrm{~min}$; gradient running time from $0 \% B$ to $100 \% B$ set to $40 \mathrm{~min}$; linear gradient and chromatogram in figure 2) was finally preferred to the program $n^{\circ} 2$ because, with equivalent efficiency, it is easier and more convenient to use a linear gradient. This program is very close to the one corresponding to the centre of the domain.

According to these chromatographic conditions, mean values and standard deviations for relative retention times of the investigated compounds were determined using five replicates giving reproducible results (Tab. VI). The linearity of the detection system was checked for each compound in a domain of 10-1000 ppm. The program was also successfully applied to qualitative and quantitative determination of phenolic products extracted from sand leachates.

In conclusion this investigation shows that the use of experimental design may be very effective to resolve weak chromatographic separations: phenolic compounds can be separated according to a relatively simple HPLC method without heating of the column and then the gradient program is therefore suitable for the analysis of these compounds in residual sands of foundry.

\section{References}

1. Hedge, J.I.; Ertel, J.R. Anal. Chem. 1982, 54, 174.

2. Kratzl, K.; Puschmann, G. Holzforschung 1960, 14, 1.

3. Stahl, E.; Schorn, P.-J. Hoppe-Seyler's Z. Physiol. Chem. 1961, 325, 263.

4. van Sumere, C.F.; Wolf, G.; Teuchy, H.; Kint, J. J. Chromatogr. 1965, 20, 48.

5. Brand, J.M. J. Chromatogr. 1966, 21, 424.

6. Hedin, P.A., Minyard. Jr., J.P.; Thompson, A.C. J. Chromatogr. 1967, 30, 43.

7. Keith, E.S.; Powers, J.J. J. Food Sci. 1966, 31, 971.

8. Dallos, F.C.; Koeppl, K.G. J. Chromatogr. Sci. 1969, 7, 565.
9. Cymbaluk, N.F.; Neudoerffer, T.S. J. Chromatogr. 1970, 51, 16.

10. Hartley, R.D. J. Chromatogr. 1971, 51, 335.

11. Hartley, R.D.; Jones, E.C. J. Chromatogr. 1975, 107, 213.

12. Vande Casteele, K.; De Pooter, H.; Van Sumere, C.F. J. Chromatogr. 1976, 121, 49.

13. Schulz, J.M.; Herrmann, K. J. Chromatogr. 1980, 195, 95.

14. Lange, H.W.; Hempel, K. J. Chromatogr. 1971, 59, 53.

15. Lange, H.W.; Männl, H.F.K.; Hempel, K. Anal. Biochem. 1970, 38, 98.

16. Andersen, J.M.; Pedersen, W.B. J. Chromatogr. 1983, 259, 131.

17. Kraak, J.C.; Huber, J.F.K. J. Chromatogr. 1974, 102, 333.

18. Terweij-Groen, C.P.; Kraak, J.C. J. Chromatogr. 1977, 138, 245.

19. Hövermann, W.; Rapp, A.; Ziegler, A. Chromatographia 1973, 6, 317.

20. Schabron, J.F.; Hurtubise, R.J.; Silver, H.F. Anal. Chem. 1978, 50, 1911.

21. Roston, D.A.; Kissinger, P.T. J. Liquid Chromatogr. 1982, 5 (suppl. 1), 75.

22. Kögel, I.; Bochter, R. Soil Biol. Biochem. 1985, 17, 637.

23. Burtscher, E.; Binder, H.; Coucin, R.; Bobleter, O. J. Chromatogr. 1982, 252, 167; ibid, 1987, 320, 401.

24. Pecina, R.; Burtscher, P. Z. Anal. Chem. 1986, 325, 461.

25. Delage, E.; Bohuon, G.; Baron, A.; Drilleau, J.F. J. Chromatogr. 1991, 555, 125.

26. Dzido, T.H.; Smolarz, H.D. J. Chromatogr. A 1994, 679, 59.

27. Guillen, D.A.; Barroso, C.G.; Perez-Bustamonte, J.A. J. Chromatogr. A 1993, 665, 227; ibid, 1996, 724, 117.

28. Barroso, C.G.; Rodriguez, M.C.; Guillen, D.A.; PerezBustamonte, J.A. J. Chromatogr. A 1996, 724, 125.

29. Glowniak, K.; Zgorka, G.; Kozyra, M. J. Chromatogr. A 1996, $730,25$.

30. Kahan, S.; Krueger, D.A. J. AOAC Int. 1997, 80, 564.

31. Andrade, P.; Ferreres, F.; Amaral, M.T. J. Liq. Chrom. \& Rel. Technol. 1997, 20, 2281.

32. Conde, E.; Cadahia, E.; Garcia-Vallejo, M.C. Chromatographia 1995, 41, 657.

33. Conde, E.; Cadahia, E.; Garcia-Vallejo, M.C.; TomasBarberan, F.T. Wood and Fiber Sci. 1995, 27, 379.

34. Benassi, M.T.; Cecchi, H.M. J. Liq. Chrom. \& Rel. Technol. 1998, 21, 491

35. Charrière, B.; Sancho, A.; Serve, L.; Combaut, G.; Gadel, F.; Piovetti, L. Journées Internationales d'Étude du Groupe Polyphénols; Montpellier, 1986, vol. 13, p 560.

36. Yost, R.W.; Ettre, L.S.; Conlon, R.D. Pratique de la chromatographie liquide; Paris: Tech. \& Doc., 1981. 